\title{
Alveolar Bone Remodeling Around Immediate Implants Placed in Accordance With the Extraction Socket Classification: A Three-Dimensional Microcomputed Tomography Analysis
}

Munirah Saleh Al-Shabeeb, * Mansour Al-Askar, ${ }^{*}$ Abdulaziz Al-Rasheed, ${ }^{*}$ Nadir Babay, ${ }^{*}$ Fawad Javed, ${ }^{* \dagger}$ Hom-Lay Wang, ${ }^{\dagger}$ and Khalid Al-Hezaimi* ${ }^{\dagger}$

Background: Previous studies assessed bone remodeling after a single tooth extraction; however, the effect of multiple contiguous teeth extractions around immediate implant remains unknown. The aim of this microcomputed tomographic investigation is to analyze the alveolar bone remodeling around immediate implants placed in accordance with the extraction socket classification (ESC).

Methods: Under general anesthesia, 10 beagle dogs underwent atraumatic tooth extractions. Animals were randomly divided into three groups, with 16 sites per group: 1) ESC-1, single tooth extraction; 2) ESC-2, two contiguous teeth extraction; and 3) ESC-3, more than two contiguous teeth extractions. Immediate implants were inserted in each socket, and postoperative plaque control measures were undertaken. After euthanasia, the jaw segments were evaluated for bone thickness, marginal bone loss (MBL), and bone-to-implant contact (BIC) using microcomputed tomography.

Results: The mean buccal bone thickness $(P<0.05)$ and MBL $(P<0.05)$ was compromised in jaws in ESC -3 compared to those in ESC-1 and ESC-2. The BIC was significantly higher among jaws in ESC - 1 compared to those in ESC-2 and ESC-3 $(P<0.05)$. There was no significant difference in the buccal bone thickness, $\mathrm{MBL}$, and BIC among the groups in the maxilla and mandible. Lingual bone remodeling did not reveal any significant differences among the groups in either jaw.

Conclusion: Buccal bone remodeling is significantly more extensive around immediate implants placed in multiple contiguous tooth extraction sites compared to immediate implants placed in single tooth extraction sites. $J$ Periodontol 2012;83:981-987.

\section{KEY WORDS}

Alveolar bone loss; bone remodeling; dental implant loading, immediate; tooth extraction; $x$-ray microtomography.

\footnotetext{
* Engineer Abdullah Bugshan Research Chair for Growth Factors and Bone Regeneration and Department of Periodontics and Community Dentistry, College of Dentistry, King Saud University, Riyadh, Saudi Arabia.

$\dagger$ Engineer Abdullah Bugshan Research Chair for Growth Factors and Bone Regeneration, 3-D Imaging and Biomechanical Lab, College of Applied Medical Sciences, King Saud University.

† Department of Periodontics and Oral Medicine, School of Dentistry, University of Michigan, Ann Arbor, MI.
}

1 he developing tooth is known to modulate the surrounding alveolar process. ${ }^{1,2}$ Bundle bone is defined as an immature bone that is supplied completely and/or solely by ligaments or tendons. At the palatal marginal crest, bundle bone is $\approx 0.2$ to $0.3 \mathrm{~mm}$ in width, whereas at the buccal marginal crest, it is frequently $\geq 2 \mathrm{~mm}$, often comprising the entire width of the buccal bone in the coronal portion of the tooth socket. ${ }^{3}$ Araújo and Lindhe ${ }^{4}$ have suggested that the buccal plate of the alveolar process comprises entirely the bundle bone.

It is well documented that tooth extraction is associated with dimensional changes in the alveolar ridge. ${ }^{3-7}$ Studies $^{3,7}$ have reported that nearly two-thirds of the alveolar bone resorption occur within the first 3 months after tooth extraction. Cardaropoli et al. ${ }^{8}$ reported that by day 14 of tooth extraction, most of the bundle bone undergoes resorption. Likewise, Nevins et al. ${ }^{5}$ demonstrated a loss of $>20 \%$ of the buccal plate of the alveolar process within the first 12 weeks of tooth extraction. Similar results were reported by Casado et al. ${ }^{9}$ and Lekovic et al. ${ }^{10}$ An explanation for these results is that, after tooth extraction, the remaining bundle bone becomes non-functional through loss

doi: 10.1902/jop.2011.110569 

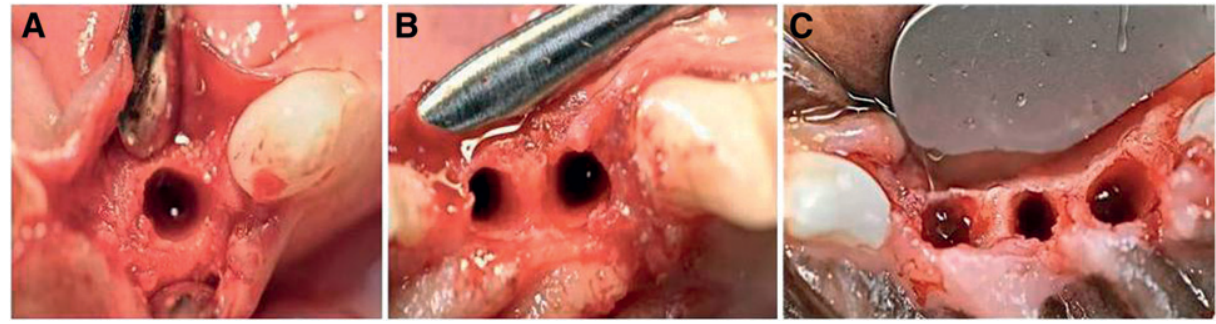

Figure I.

Sample extraction sites. A) ESC-I; B) ESC-2; and C): ESC-3. Evidence of sound buccal bone with no dehiscence.
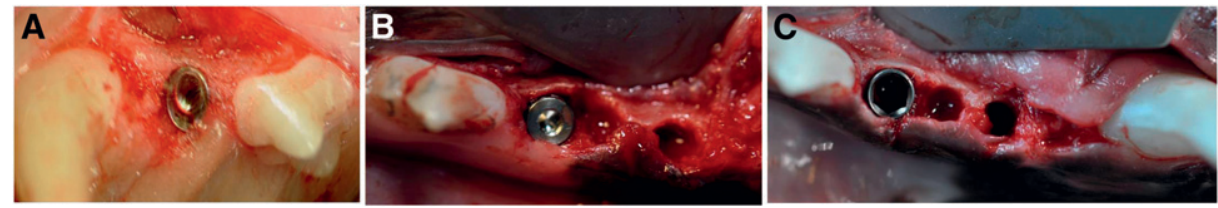

Figure 2.

Immediate implants submerged in bone: $\boldsymbol{A})$ ESC-I; B) ESC-2; and C) ESC-3.

tractions. The present study is based on the hypothesis that immediate implants placed in multiple contiguous teeth extraction sites display a more intense alveolar bone remodeling compared to implants placed in single tooth extraction sites. The aim of this three-dimensional microcomputed tomographic analysis is to investigate the alveolar bone remodeling around immediate implants placed in single tooth and contiguous teeth extraction sites.

\section{MATERIALS AND METHODS}

\section{Ethics Approval and Study Animals}

The study protocol was approved by the ethics committee at the College of Dentistry,

of its blood supply from the periodontal ligament and ultimately undergoes complete resorption. ${ }^{4}$

Various treatment strategies have been proposed to reduce bone resorption after tooth extraction. ${ }^{11-13}$ Some studies ${ }^{14-17}$ have reported that immediate placement of dental implants after tooth extraction plays an essential role in reducing alveolar bone resorption particularly on the buccal aspect of the alveolar process. Covani et al. ${ }^{16}$ investigated the physiologic bone remodeling in beagle dogs after the placement of immediate implants in fresh extraction sites and concluded that this contributes to reducing the rate of alveolar buccal bone resorption; ${ }^{16}$ however, controversial results have also been reported. In a study on beagle dogs, Araújo et al. ${ }^{17}$ reported that the placement of an immediate implant in the fresh extraction sites does not prevent alveolar bone remodeling. A study by van Kesteren et al. ${ }^{18}$ found no significant differences between patients treated with immediate or delayed approaches for mid-buccal or interproximal soft-tissue margins. It is notable that these reports ${ }^{16-18}$ were based on results obtained only after single tooth extraction.

In a study on monkeys, Al-Hezaimi et al. ${ }^{19}$ proposed the following extraction socket classification (ESC): 1) Class 1: single tooth extraction; 2) Class 2: two contiguous teeth extractions; and 3) Class 3: $>2$ contiguous teeth extractions. This study ${ }^{19}$ highlighted the histologic finding that the buccal bone also receives a blood supply from the adjacent interdental bone and not solely from the socket side of the alveolus. Recently, Al-Askar et al. ${ }^{20}$ confirmed that contiguous tooth extraction resulted in significiantly more alveolar bone resorption than single tooth ex-
King Saud University, Riyadh, Saudi Arabia. Ten healthy adult female beagle dogs(mean age, $24 \pm$ 0.83 months; weight, $13.8 \pm 0.49 \mathrm{~kg}$ ) were used. The animals were kept in individual cages and vaccinated against rabies and infectious hepatitis.

\section{Animal Grouping and Surgical Protocol}

The animals were randomly divided into three groups ${ }^{19}$ by picking a paper marked "Group 1" (ESC-1), "Group 2" (ESC-2), or "Group 3" (ESC-3) from a brown bag. In ESC-1 (two dogs), 16 bilateral maxillary and mandibular first and third premolars were extracted and replaced with immediate implants. In ESC-2 (four dogs), two adjacent premolars were extracted in 16 posterior sites (maxillary and mandibular) with one immediate implant placement in the mesial socket in the upper arch and distal socket in the lower arch to avoid contacting the root of the distally curved canine root in the lower arch. In ESC-3 (four dogs), three teeth were extracted in 16 posterior sites (maxillary and mandibular) with immediate implant placement in the middle of the extraction sites (Fig. 1).

Preoperatively, the dogs received supragingival scaling, twice weekly for 3 weeks using an ultrasonic scaler.\$ Intramuscular amoxicillin $(25 \mathrm{mg} / \mathrm{kg}$ body weight) was administered on the day of surgery. The non-surgical and surgical procedures were performed under general anesthesia using ketamine ${ }^{\mathbb{I}}(10 \mathrm{mg} / \mathrm{kg}$ body weight) and local anesthesia with lidocaine ${ }^{\#}$ (with

\footnotetext{
$\S$ Hu-Friedy, Chicago, IL.

I| Betamox LA, Norbrook Laboratory, Newry, County Down, Northern Ireland.

Il Pfizer, Sandwich, Kent, UK.

\# Xylocaine, AstraZeneca for DENTSPLY Pharmaceutical, York, PA.
} 
Table I.

\section{Buccal Bone Thickness, MBL, and BIC in Groups 1, 2 and 3}

\begin{tabular}{|c|c|c|c|}
\hline & Group I (ESC-I) & Group 2 (ESC-2) & Group 3 (ESC-3) \\
\hline $\begin{array}{l}\text { Buccal bone thickness }(\mathrm{mm}) \\
\quad(\text { mean } \pm \mathrm{SD})\end{array}$ & $1.40 \pm 0.41^{* \neq}$ & $0.84 \pm 0.64 * *^{\dagger}$ & $0.28 \pm 0.18^{\dagger \ddagger}$ \\
\hline $\mathrm{MBL}(\mathrm{mm})($ mean $\pm \mathrm{SD})$ & $0.375 \pm 0.108 \rrbracket$ & $2.00 \pm 0.30 \S \|$ & $3.20 \pm 0.80 \| \boldsymbol{\|}$ \\
\hline $\mathrm{BIC}($ mean \%) & $79.29 \pm 8.0^{\# \dagger \dagger}$ & $57.21 \pm 6.31^{\# * *}$ & $47.14 \pm 7.69 * * \dagger \dagger$ \\
\hline
\end{tabular}

* $P<0.05$

$\dagger P<0.001$.

† $P<0.001$.

$\S P<0.05$.

$P<0.05$.

If $P<0.001$.

$\# P<0.001$.

$* * P<0.05$.

$\dagger \uparrow P<0.001$.

$5 \mathrm{mg} / \mathrm{mL}$ epinephrine). Under general anesthesia, an electric saw** and forceps were used for extractions. The animals were draped, and the surgical site was swabbed with povidone-iodine solution. ${ }^{\dagger \dagger}$

Using a sulcular incision (\#15 blade), full-thickness buccal flaps were raised and extended to the mucogingival junction. The teeth were extracted atraumatically using a an electric saw. ${ }^{\ddagger}$ None of the extracted teeth experienced dehiscence (Fig. 1). A total of 48 immediate-implant fixtures $\S \S$ (10.5 mm long; $3 \mathrm{~mm}$ diameter) were placed (16 fixtures ${ }^{\| I}$ per group) (Fig. 2). All fixtures If $^{\text {w }}$ were inserted in the center of the extraction socket in a submerged position leaning toward the lingual side, and healing screws were placed. A minimum of $1.5 \mathrm{~mm}$ distance was maintained between each implant and the adjacent tooth. The average gap between the implant and extraction socket in each group was $0.75 \mathrm{~mm}$. Flaps were sutured using polyglactin 910 sutures $^{\# \#}$ and primary closure was achieved.

\section{Postoperative Management}

All animals received intramuscular injections of amoxicillin*** ( $5 \mathrm{mg} / \mathrm{kg}$ body weight daily for 3 days), and plaque control procedures, which included topical application of a $0.2 \%$ chlorhexidine digluconate solution, ${ }^{\dagger \dagger}$ were performed twice weekly for 4 months post-surgery. After 2 weeks, the sutures were removed, and periapical radiographs were taken to assess the relationship of the implants with the adjacent vital structures. Intramuscularly administered antibiotics ${ }^{\ddagger} \neq$ were continued for 3 days post-surgery at 25 to $50 \mathrm{mg} / \mathrm{kg}$ every 8 hours.

The animals were sacrificed after 4 months with an overdose of $3 \%$ sodium pentobarbital.

\section{Hard-Tissue Sectioning and Microcomputed Tomography Analysis}

The jaw segments containing the dental implants, adjacent mesial and distal teeth, and alveolar bone struc- tures were removed en bloc using an electric saw§§§ and fixed in $10 \%$ neutral formalin solution. The segments were analyzed using a three-dimensional microcomputed tomography scan. the unit was operated at an accelerated potential of $101 \mathrm{~kg} / \mathrm{V}$ with a beam current of $96 \mu \mathrm{A}$ using an aluminum filter with a resolution of $37.41 \mu \mathrm{m}$ pixels. In both arches, the buccal and lingual bone thickness and bone-to-implant contacts (BICs) were measured.

\section{Measurement of Buccal Bone Thickness Using Microcomputed Tomography}

Bone thickness was determined as horizontal distance from the crest of the implant to the bone crest at the buccal and lingual aspects of the implant. The measurement was performed at six different axial points at 1 -mm intervals from the coronal to the apical part of the implant.

\section{Measurement of Marginal Bone Loss and BIC}

The marginal bone loss (MBL) around the implants was measured by drawing a horizontal line between cemento-enamel junctions of teeth adjacent to the implant and then measuring the vertical bone height from the line until the bone crest. ${ }^{21}$

The BIC was calculated using software. "qा? The analysis software assessed the total surface area of the region of interest and the subset of the region-of-interest surface that is intersected by binarized bone objects. ${ }^{22}$ The parameter thus measured was termed "intersection surface," which corresponded to the BIC. The BIC was

\footnotetext{
* * Piezosurgery, Mectron Medical Technology, Columbus, $\mathrm{OH}$.

$\dagger \dagger$ Betadine, The Purdue Fredrick Company, Stamford, CT.

邦 Piezosurgery, Mectron Medical Technology.

$\S \S$ Laser-Lok Microchannels, BioHorizons, Birmingham, AL.

III Laser-Lok Microchannels, BioHorizons.

II Laser-Lok Microchannels, BioHorizons.

\#\# VICRYL sutures, Ethicon, Johnson \& Johnson, Somerville, NJ.

*** Betamox LA, Norbrook Laboratory.

$\dagger \dagger \dagger$ GUM, Sunstar Americas, Chicago, IL.

$\neq \neq \neq$ Betamox LA, Norbrook Laboratory.

$\S \S \S$ GUM, Sunstar Americas,

|ll|| Leica SP1600, Leica Microsystems, Bannockburn, IL.

ๆๆ
} 
calculated as the percentage of implant surface in contact with the bone through the whole perimeter of the implant at $\times 100$ magnification. ${ }^{22}$

\section{Statistical Analysis}

Data were analyzed using a statistical software program."\#\# Differences between the buccal and lingual bone thickness, MBL, and BIC among the three groups were assessed using one-way analysis of variance. $P<0.05$ was considered statistically significant.

\section{RESULTS}

All implants $(n=48)$ healed uneventfully, and bone remodeling was observed in all groups 4 months after implant placement.

\section{Bone Thickness}

There was a significant difference in the mean buccal bone thickness among ESC-1 (1.40 \pm 0.41$)$, ESC-2 $(0.84 \pm 0.64)$, and ESC-3 $(0.28 \pm 0.18)$, as shown in Table 1. There was no significant difference in the lingual bone thickness among the three groups.

In ESC-1, one case displayed implant thread exposure on the buccal surface, but none was observed on the lingual/palatal surface. In ESC-1, bone was present at the smooth surface of the implant in all the cases (Figs. 3A, 3B, and 4A).

In ESC-2, buccal bone remodeling was significantly increased compared to ESC-1 $(P<0.05)$. In this group, an average of two implant threads was exposed on the buccal side, but again there was no exposure to the lingual/palatal surface (Figs. 3C, 3D, and 4B).

In ESC-3, a significantly pronounced buccal bone remodeling was observed compared to ESC-1 $(P<0.001)$ and ESC $-2(P<0.05)$. In this group, an average of three to four implant threads were exposed on the buccal side and on the lingual/palatal sides (Figs. 3E, 3F, and 4C).

In all three groups, there was no significant difference in bone remodeling around implants placed in the maxilla and mandible.

\section{$M B L$}

MBL was observed in all groups after placement of the implants. The highest MBL was noticed in ESC-3 $(3.20 \pm 0.80)$ compared to ESC-2 (2.00 \pm 0.30$)$ $(P<0.05)$ and ESC-1 $(0.375 \pm 0.10)(P<0.001)$. The resorption primarily occurred on the buccal surface, and there was no significant resorption on the interdental and lingual/palatal bone. The MBL mesial and distal to the implants is shown in Table 2.

\section{$B I C$}

There was a statistically significant difference in the $\mathrm{BIC}$ among the three groups. The highest BIC was observed in ESC-1 (mean BIC, 79.7\%) compared to ESC-2 (mean BIC, 55.3\%) $(P<0.05)$ and ESC-3 (mean BIC, 49.4\%) $(P<0.001)$.

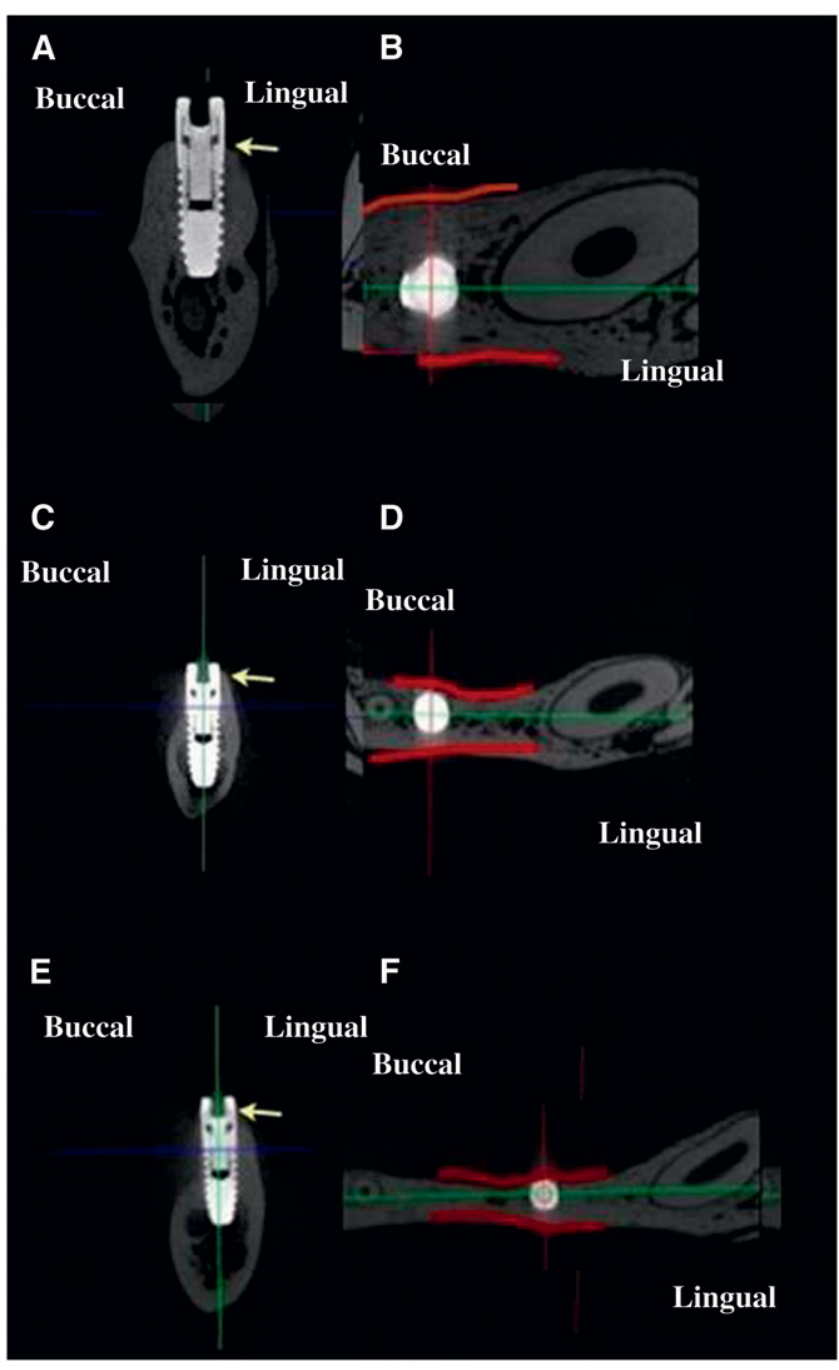

Figure 3.

A series of reconstructed sagittal and axial microcomputed tomography images illustrating the measurements of the buccal bone thickness and volume of bone around immediate implant. $\boldsymbol{A}, \boldsymbol{C}$, and $\mathbf{E})$ Sagittal sections show the buccal and lingual bone thickness around the implant placed in ESC-1,-2, and -3, respectively. Arrows show that the lingual bone is at the smooth surface of the implant. $\mathbf{B}, \mathbf{D}$, and $\mathbf{F}$ ) Axial section at the level of the crestal bone showing the different buccal bone thickness around dental implants at the crestal bone level in ESC-1, -2, and -3, respectively. Red line shows the border of the buccal and lingual bone.

\section{DISCUSSION}

This study shows that extraction of contiguous multiple teeth is associated with significantly more buccal bone remodeling around immediate implants compared to implants placed in single tooth extraction sites. This is in agreement with the studies that emphasized the importance of the interdental bone blood supply to the buccal bone. ${ }^{19,20}$

Bone remodeling is a complex process in which old bone is removed by the process of resorption and is

\#\#\# SkyScan 1172 CT-Analyser, version 1.11.4.2+, Skyscan, Kontich Belgium. 


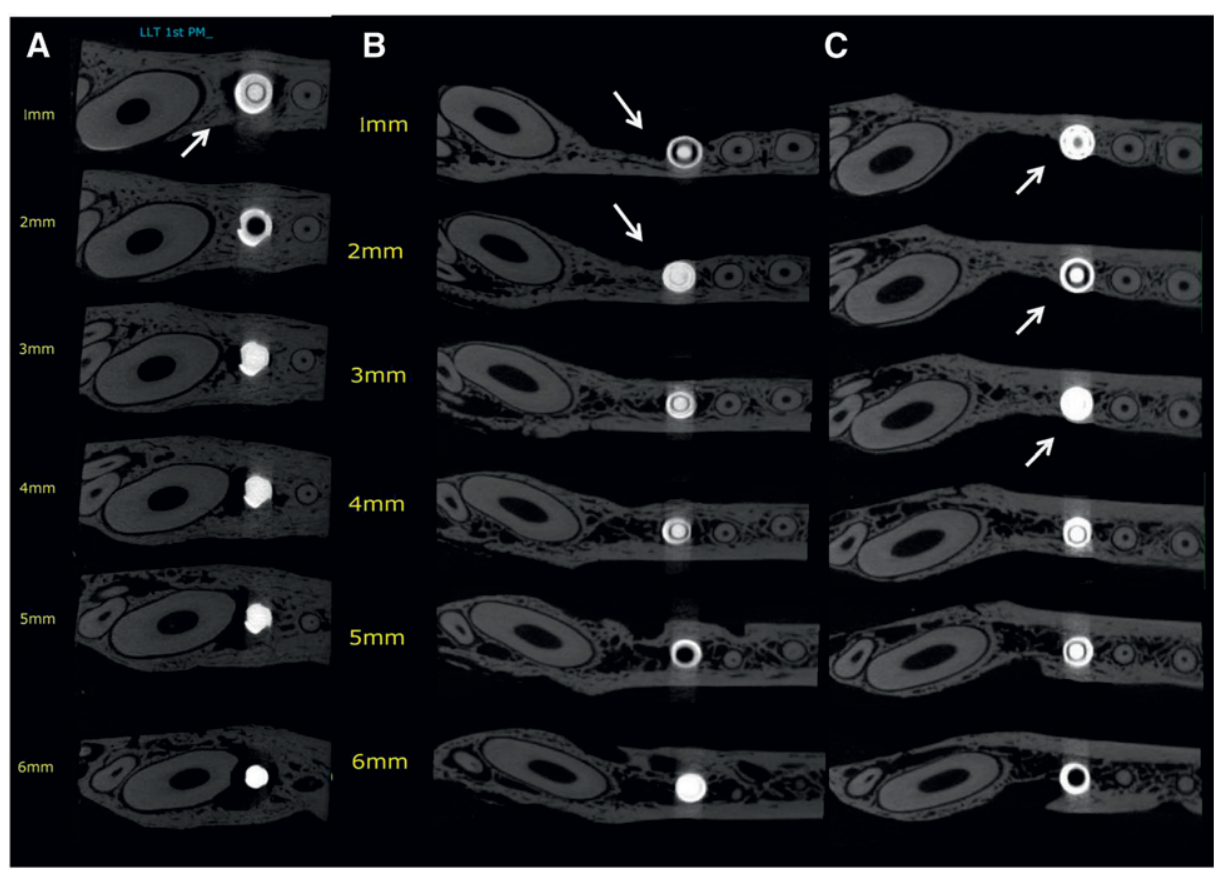

Figure 4.

A series of reconstructed axial images illustrating the buccal and lingual bone thickness at six different sections of I-mm intervals starting I mm below the cemento-enamel junction (arrows indicate the buccal side). $\boldsymbol{A}$ ) Evidence of buccal bone presence at the first I-mm axial section to the apex of the implant. There was no significance difference in the mean of buccal bone thickness between different sections. B) Illustrates the absence of the buccal bone in the first $2 \mathrm{~mm}$. C) Illustrates the absence of the buccal bone in the first $3 \mathrm{~mm}$ and presence of very thin buccal bone buccal bone demonstrating very thin bone thickness at $4 \mathrm{~mm}$ compared to the thickness at $6 \mathrm{~mm}$ (arrows at 3,2, and I mm). In all sections in all different classes, there was no significant difference in the lingual bone thickness.

Table 2.

\section{Mean MBL Around Implants in Each Group}

\begin{tabular}{lcl}
\hline ESC & $\begin{array}{c}\text { Mesial to Implant }(\mathrm{mm}) \\
\text { (mean } \pm \mathrm{SD})\end{array}$ & $\begin{array}{c}\text { Distal to Implant }(\mathrm{mm}) \\
\text { (mean } \pm \mathrm{SD})\end{array}$ \\
\hline Group 1 & $0.37 \pm 0.11^{*}$ & $0.38 \pm 0.10^{*}$ \\
Group 2 & $1.61 \pm 0.65^{\dagger}$ & $2.06 \pm 0.43$ \\
Group 3 & $2.70 \pm 0.82^{* \dagger}$ & $2.76 \pm 0.86^{*}$ \\
\hline
\end{tabular}

* $P<0.05$, comparison between Groups 1 and 3 .

$\dagger P<0.05$, comparison between Groups 2 and 3 .

replaced by new bone. It has been suggested that, after tooth extraction, the blood vessels to the bone walls are severed, thereby causing bundle bone resorption; ${ }^{4}$ however, other reports ${ }^{19,20}$ have shown that the interdental bone blood supply to the buccal bone is also a significant factor in this regard. This loss of blood supply to the bone may cause death of the osteocytes and necrosis of the surrounding mineralized tissue. Thus, it is likely that extraction of contiguous teeth severs the blood vessels of the alveolar socket and also interdental bone blood vessels, thereby diminishing the vascular supply to the alveo- lar socket. In the present study, a limited flap to the mucogingival junction was used during implant placement in all groups and the only variable was the number of teeth extracted.

Covani et al. ${ }^{16}$ demonstrated that immediate placement of dental implants into fresh extraction sockets helps reduce the rate of buccal bone resportion after tooth extraction; however, controversial results have also been reported. ${ }^{17,18}$ Araujo et al. ${ }^{17}$ and van Kesteren et al. ${ }^{18}$ reported that placement of an implant in the fresh extraction site does not prevent alveolar bone remodeling that occurred in the walls of the socket. It is notable that, in the study by Covani et al., ${ }^{16}$ the distal roots of third and the fourth premolars were hemisectioned and immediate implants were placed in the fresh single extraction sites. According to Al-Hezaimi et al., ${ }^{19}$ the extraction socket may still be receiving blood supply from the adjacent interdental bone, thereby reducing the rate of buccal bone resorption. The present study supports the results by Covani et al. ${ }^{16}$ to an extent, because immediate implants placed in a single tooth extraction site (ESC-1) showed slight buccal bone loss compared to immediate implants installed in sites with multiple contiguous teeth extractions (ESC-2 and ESC-3). ${ }^{20}$ With reference to jaw location (either maxilla or mandible), no significant difference in alveolar bone remodeling around immediate implants**** were observed in any group. This suggests that alveolar bone remodeling around immediate implants placed in fresh extraction sites occurs in a standardized pattern, and the role of bone morphology surrounding the fixture is rather secondary; however, additional studies are warranted in this regard. The present

**** Laser-Lok Microchannels, BioHorizons. 
results may provide a platform for clinicians when to place an implant and to consider the patterns of buccal bone remodeling that may occur in the patients with contiguous multiple extractions that are scheduled for implant placement.

A recent histologic study on monkeys emphasized the significance of the interdental bone blood supply to the buccal bone. ${ }^{19}$ This study ${ }^{19}$ demonstrated that trauma to the vascular supply of the interdental bone is directly proportional to the number of teeth extracted. Similar results were reported by Al-Askar et al. ${ }^{20}$ The present results support these studies ${ }^{19,20}$ as a significantly higher mesial and distal MBL was observed around immediate implants placed in ESC-3 compared to those placed in the other groups. However, our results are in contradiction to previous studies $^{15,16,23}$ that reported that immediate placement of dental implants in fresh extraction sites reduces buccal bone remodeling and MBL.

The implant surface morphology has been reported to influence the overall peri-implant hard- and softtissue morphology. ${ }^{24}$ The present study may offer possible buccal bone remodeling patterns after different extraction sockets associated with immediate implants. The present results showed that the lingual/ palatal bone was at the smooth surface of the lasermicroetched implant. In a recent study on dogs, Nevins et al. ${ }^{25}$ investigated the effectiveness of laser-ablated microgrooves placed within implant collars in supporting direct connective tissue attachments. The results showed significant improvement in peri-implant hardand soft-tissue healing around laser-microetched implants compared to the traditional machined abutment surfaces.

\section{CONCLUSION}

Immediate implants placed in contiguous teeth extraction sites experience more extensive buccal bone remodeling compared to immediate implants placed in single tooth extraction sites.

\section{ACKNOWLEDGMENTS}

The authors report no conflicts of interest related to this study.

\section{REFERENCES}

1. Odajima T. A longitudinal study on growth and development of dental arches of primary, mixed and permanent dentitions (in Japanese).Shikwa Gakuho 1990;90:369-409.

2. Machida Y. Growth and development of the alveolar ridge after extraction of deciduous teeth and its clinical application (in Japanese).Shoni Shikagaku Zasshi 1989;27:587-594.

3. Schropp L, Wenzel A, Kostopoulos L, Karring T. Bone healing and soft tissue contour changes following single-tooth extraction: A clinical and radiographic 12-month prospective study. Int $J$ Periodontics Restorative Dent 2003;23:313-323.

4. Araújo MG, Lindhe J. Dimensional ridge alterations following tooth extraction. An experimental study in the dog. J Clin Periodontol 2005;32:212-218.

5. Nevins M, Camelo M, De Paoli S, et al. A study of the fate of the buccal wall of extraction sockets of teeth with prominent roots. Int $J$ Periodontics Restorative Dent 2006;26:19-29.

6. Araújo MG, Wennström JL, Lindhe J. Modeling of the buccal and lingual bone walls of fresh extraction sites following implant installation. Clin Oral Implants Res 2006; 17:606-614.

7. Schropp L, Wenzel A, Kostopoulos L. Impact of conventional tomography on prediction of the appropriate implant size. Oral Surg Oral Med Oral Pathol Oral Radiol Endod 2001;92:458-463.

8. Cardaropoli G, Araújo M, Lindhe J. Dynamics of bone tissue formation in tooth extraction sites. An experimental study in dogs. J Clin Periodontol 2003;30:809-818.

9. Casado PL, Duarte ME, Carvalho W, Esmeraldo da Silva L, Barboza EP. Ridge bone maintenance in human after extraction. Implant Dent 2010;19:314-322.

10. Lekovic V, Camargo PM, Klokkevold PR, et al. Preservation of alveolar bone in extraction sockets using bioabsorbable membranes. J Periodontol 1998;69: 1044-1049.

11. Kim DM, Nevins $M$, Camelo $M$, et al. The feasibility of demineralized bone matrix and cancellous bone chips in conjunction with an extracellular matrix membrane for alveolar ridge preservation: A case series. Int $J$ Periodontics Restorative Dent 2011;31:39-47.

12. Fiorellini JP, Nevins ML. Localized ridge augmentation/preservation. A systematic review. Ann Periodontol 2003;8:321-327.

13. Wang HL, Kiyonobu K, Neiva RF. Socket augmentation: Rationale and technique. Implant Dent 2004;13: 286-296.

14. Lazzara RJ. Immediate implant placement into extraction sites: Surgical and restorative advantages. Int $J$ Periodontics Restorative Dent 1989;9:332-343.

15. Vignoletti $F$, de Sanctis $M$, Berglundh T, Abrahamsson I, Sanz M. Early healing of implants placed into fresh extraction sockets: An experimental study in the beagle dog. II: Ridge alterations. J Clin Periodontol 2009;36:688-697.

16. Covani U, Cornelini R, Calvo JL, Tonelli P, Barone A. Bone remodeling around implants placed in fresh extraction sockets. Int $J$ Periodontics Restorative Dent 2010;30:601-607.

17. Araújo MG, Sukekava F, Wennström JL, Lindhe J. Ridge alterations following implant placement in fresh extraction sockets: An experimental study in the dog. $J$ Clin Periodontol 2005;32:645-652.

18. van Kesteren CJ, Schoolfield J, West J, Oates T. A prospective randomized clinical study of changes in soft tissue position following immediate and delayed implant placement. Int $J$ Oral Maxillofac Implants 2010;25:562-570.

19. Al-Hezaimi K, Levi P, Rudy R, Al-Jandan B, AlRasheed A. An extraction socket classification developed using analysis of bone type and blood supply to the buccal bone in monkeys. Int $J$ Periodontics Restorative Dent 2011;31:421-427.

20. Al-Askar M, O'Neill R, Stark P, Griffin T, Javed F, AlHezaimi K. Effect of single and contiguous teeth extractions on alveolar bone remodeling: A study in 
dogs [published online ahead of print December 15, 2011]. Clin Implant Dent Relat Res. doi: 10.1111/j. 1708-8208.2011.00403.x.

21. Ishikawa $T$, Salama $M$, Funato $A$, et al. Threedimensional bone and soft tissue requirements for optimizing esthetic results in compromised cases with multiple implants. Int $J$ Periodontics Restorative Dent 2010;30:503-511.

22. Salmon P. Micro-CT 3D image analysis techniques for orthopedic applications: Metal implant-to-bone contact surface and porosity of biomaterials. In: Leung K-S, Qin l, Cheung WH, eds.A Practical Manual for Musculoskeletal Research. Singapore: World Scientific Publishing; 2008;583-604.

23. Park YS, Lee SP, Han CH, Kwon JH, Jung YC. The microtomographic evaluation of marginal bone resorption of immediately loaded scalloped design implant with various microthread configurations in canine mandible: Pilot study. J Oral Implantol 2010;36:357-362.
24. Javed F, Almas K, Crespi R, Romanos GE. Implant surface morphology and primary stability: Is there a connection? Implant Dent 2011;20:40-46.

25. Nevins M, Kim DM, Jun SH, Guze K, Schupbach P, Nevins ML. Histologic evidence of a connective tissue attachment to laser microgrooved abutments: A canine study. Int J Periodontics Restorative Dent 2010; 30:245-255.

Correspondence: Dr. Khalid Al-Hezaimi, Engineer Abdullah Bugshan Research Chair for Growth Factors and Bone Regeneration, 3D Imaging and Biomechanical Lab, College of Applied Medical Sciences; and Department of Periodontics and Community Dentistry, College of Dentistry, King Saud University, P.O. Box 60169, Riyadh 11545, Saudi Arabia. E-mail: hezaimik16@gmail.com.

Submitted September 24, 2011; accepted for publication November 2, 2011. 